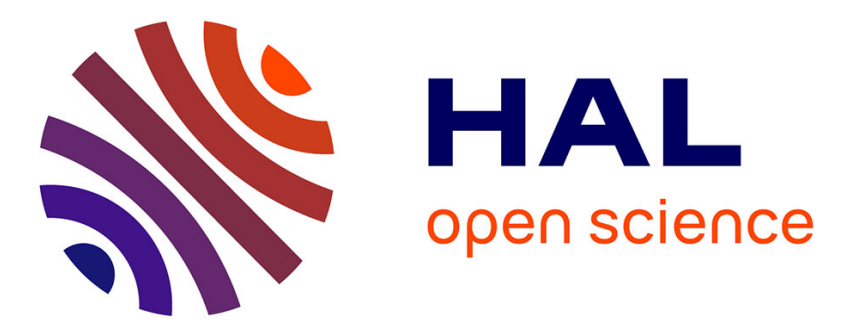

\title{
Guaranteed nonlinear estimation using constraint propagation on sets
}

\author{
Luc Jaulin, Michel Kieffer, Isabelle Braems, Eric Walter
}

\section{To cite this version:}

Luc Jaulin, Michel Kieffer, Isabelle Braems, Eric Walter. Guaranteed nonlinear estimation using constraint propagation on sets. International Journal of Control, 2001, 74 (18), pp.1772-1782. hal00845164

\section{HAL Id: hal-00845164 \\ https://hal.science/hal-00845164}

Submitted on 16 Jul 2013

HAL is a multi-disciplinary open access archive for the deposit and dissemination of scientific research documents, whether they are published or not. The documents may come from teaching and research institutions in France or abroad, or from public or private research centers.
L'archive ouverte pluridisciplinaire HAL, est destinée au dépôt et à la diffusion de documents scientifiques de niveau recherche, publiés ou non, émanant des établissements d'enseignement et de recherche français ou étrangers, des laboratoires publics ou privés. 


\title{
Guaranteed nonlinear estimation using constraint propagation on sets
}

\author{
L. Jaulin ${ }^{12}$, M. Kieffer ${ }^{1}$, I. Braems ${ }^{1}$ and E. Walter ${ }^{1}$
}

\begin{abstract}
Bounded-error estimation is the estimation of the parameter or state vector of a model from experimental data, under the assumption that some suitably defined errors should belong to some prior feasible sets.

When the model outputs are linear in the vector to be estimated, a number of methods are available to enclose all estimates that are consistent with the data into simple sets such as ellipsoids, orthotopes or parallelotopes, thereby providing guaranteed set estimates.

In the nonlinear case, the situation is much less developed and there are very few methods that produce such guaranteed estimates. In this paper, the discrete-time problem is cast into the more general framework of constraint satisfaction problems. Algorithms rather classical in the area of interval constraint propagation are extended by replacing intervals by more general subsets of real vector spaces. This makes it possible to propose a new algorithm that contracts the feasible domains for each uncertain variable optimally (i.e., no smaller domain could be obtained) and efficiently.

The resulting methodology is illustrated on discrete-time nonlinear state estimation. The state at time $k$ is estimated either from past measurement only or from all measurements assumed to be available from the start. Even in the causal case, prior information on the future value of the state and output vectors, due for instance to physical constraints, is readily taken into account.
\end{abstract}

Keywords: bounded-error estimation, constraint propagation, CSP, identification, interval analysis, nonlinear estimation, observation, set estimation.

\section{Introduction}

In a linear context, many tools are available to estimate the parameter or state vector of a model from experimental data. They can be classified according to how they deal with uncertainty. Some of them do not take explicitly into account the fact that the model is an approximation

\footnotetext{
${ }^{1}$ Laboratoire des Signaux et Systèmes, UMR 8506, CNRS-Supélec-Université de Paris Sud, 91192 Gif-surYvette, France

${ }^{2}$ on leave from Laboratoire d'Ingénierie des Systèmes Automatisés, Université d'Angers, France.
} 
of reality and that the measurements are corrupted by noise. This is the case, for instance, of Luenberger state observers [14] and of many adaptive schemes [13]. Other estimators, such as maximum-likelihood estimators [9] or the ubiquitous Kalman filter [7] [20], are based on a statistical description of uncertainty, and assume that the measurement noise and state perturbations are realizations of random variables, with known statistical properties. A last group of methods, known under the generic names of set-membership estimation or guaranteed estimation or bounded-error estimation, see, e.g., [21], [18], [19], [15] and the references therein, is based on the assumption that the uncertain variables all belong to known compact sets, and attempts to build simple sets, such as ellipsoids, orthotopes or parallelotopes, guaranteed to contain the vectors to be estimated.

In a nonlinear context, the methodology is far less developed, and still the subject of active research even in the deterministic case [8]. When uncertainty is explicitly taken into account, this is usually by resorting to linearization. For parameter estimation, one may exploit the asymptotic properties of maximum-likelihood estimators, but the validity of the results obtained from a short data base is then questionable. For state estimation, an extended Kalman filter [4], based on a linearization of the model around its trajectory is usually employed. This linearization is inherently local and may fail to produce reliable estimates. It makes any statistical interpretation of the covariance matrices computed by the algorithm questionable, because the propagation of the statistical properties of the perturbations through the nonlinear system is largely unknown. As far as set membership estimation is concerned, very few guaranteed methods are available, most of them developed for parameter estimation. They are based on branch-and-bound techniques (see, e.g., [16] for a signomial programming approach and [6] for an interval computation approach). A method based on interval analysis to compute guaranteed state estimates was proposed in [11] and [12].

The purpose of this paper is to present a new approach for the guaranteed estimation of the parameter and/or state vector of a nonlinear discrete-time model in a bounded-error context. Consider a nonlinear discrete-time system described by

$$
\left\{\begin{array}{l}
\mathbf{x}_{k}=\mathbf{f}_{k}\left(\mathbf{x}_{k-1}, \mathbf{p}, \mathbf{w}_{k-1}, \mathbf{u}_{k-1}\right) \\
\mathbf{y}_{k}=\mathbf{g}_{k}\left(\mathbf{x}_{k}, \mathbf{p}, \mathbf{w}_{k}, \mathbf{u}_{k}\right)
\end{array} \quad k=1, \ldots, \bar{k},\right.
$$

where $k$ is the time index, $\mathbf{x}_{k}$ is the state vector, $\mathbf{y}_{k}$ is the output vector, $\mathbf{u}_{k}$ is the input vector, $\mathbf{w}_{k}$ is the perturbation vector, $\mathbf{p}$ is a constant parameter vector and $\mathbf{f}_{k}$ and $\mathbf{g}_{k}$ are known functions. The set of all variables involved in this problem is

$$
\mathcal{V}=\left\{\mathbf{p}, \mathbf{x}_{0}, \mathbf{w}_{0}, \mathbf{u}_{0}, \mathbf{x}_{1}, \mathbf{w}_{1}, \mathbf{u}_{1}, \mathbf{y}_{1}, \ldots, \mathbf{x}_{\bar{k}}, \mathbf{w}_{\bar{k}}, \mathbf{u}_{\bar{k}}, \mathbf{y}_{\bar{k}}\right\}
$$

We shall assume that there exist some unknown actual values, denoted by $\mathbf{x}_{k}^{*}, \mathbf{w}_{k}^{*}, \mathbf{u}_{k}^{*}, \mathbf{y}_{k}^{*}$ and $\mathbf{p}^{*}$, for $\mathbf{x}_{k}, \mathbf{w}_{k}, \mathbf{u}_{k}, \mathbf{y}_{k}$ and $\mathbf{p}$, such that (1) is satisfied. This assumption will allow us to interpret 
the estimation problem as that of finding reliable estimates for these actual values, but note that it is not required for the application of the method.

The set-membership approach to be followed in this paper characterizes the uncertainty about the actual value $\mathbf{v}^{*}$ of any given variable $\mathbf{v} \in \mathcal{V}$ by associating with $\mathbf{v}$ a domain $\mathbb{V}$ that contains $\mathbf{v}^{*}$. The set of all such domains is

$$
\mathcal{D}=\left\{\mathbb{P}, \mathbb{X}_{0}, \mathbb{W}_{0}, \mathbb{U}_{0}, \mathbb{X}_{1}, \mathbb{W}_{1}, \mathbb{U}_{1}, \mathbb{Y}_{1}, \ldots, \mathbb{X}_{\bar{k}}, \mathbb{W}_{\bar{k}}, \mathbb{U}_{\bar{k}}, \mathbb{Y}_{\bar{k}}\right\}
$$

When the actual value of a variable $\mathbf{v}$ is known exactly, $\mathbb{V}$ is the singleton $\left\{\mathbf{v}^{*}\right\}$. When nothing is known about $\mathbf{v}^{*}, \mathbb{V}=\mathbb{R}^{\operatorname{dim} \mathbf{v}}$.

A measurement $\mathbf{v}^{\mathrm{m}}$ of a variable $\mathbf{v} \in \mathcal{V}$ provides an approximation of $\mathbf{v}^{*}$. Let $\mathcal{P}\left(\mathbb{R}^{\operatorname{dim} \mathbf{v}}\right)$ be the set of all subsets of $\mathbb{R}^{\text {dim } \mathbf{v}}$. We shall call interpretation function associated with $\mathbf{v}$, a setvalued function $\phi_{\mathbf{v}}: \mathbb{R}^{\operatorname{dim} \mathbf{v}} \rightarrow \mathcal{P}\left(\mathbb{R}^{\operatorname{dim} \mathbf{v}}\right)$ that satisfies $\mathbf{v}^{*} \in \phi_{\mathbf{v}}\left(\mathbf{v}^{\mathrm{m}}\right)$. The set $\phi_{\mathbf{v}}\left(\mathbf{v}^{\mathrm{m}}\right)$ is the measurement uncertainty set. As soon as $\mathbf{v}^{\mathrm{m}}$ is made available, the domain $\mathbb{V}$ for $\mathbf{v}$ can be replaced by $\mathbb{V} \cap \phi_{\mathbf{v}}\left(\mathbf{v}^{\mathrm{m}}\right)$. In (1), only the variables $\mathbf{u}_{k}$ and $\mathbf{y}_{k}$ are assumed to be measured. Two situations will be distinguished:

- A causal context: at time $k$, the measurements are available up to time $k$ only, i.e., the available data are $\left\{\mathbf{u}_{0}^{\mathrm{m}}, \mathbf{u}_{1}^{\mathrm{m}}, \mathbf{y}_{1}^{\mathrm{m}}, \ldots, \mathbf{u}_{k}^{\mathrm{m}}, \mathbf{y}_{k}^{\mathrm{m}}\right\}$.

- A noncausal context: all measurements $\left\{\mathbf{u}_{0}^{\mathrm{m}}, \mathbf{u}_{1}^{\mathrm{m}}, \mathbf{y}_{1}^{\mathrm{m}}, \ldots, \mathbf{u}_{k}^{\mathrm{m}}, \mathbf{y}_{k}^{\mathrm{m}}, \ldots, \mathbf{u}_{\bar{k}}^{\mathrm{m}}, \mathbf{u}_{\bar{k}}^{\mathrm{m}}\right\}$ are available from the start.

This distinction is similar to that between estimating and smoothing Kalman filters. Even in a causal context, some prior information may be available on variables before any measurement is collected. For instance, physical constraints may provide upper and lower bounds on some components of vector variables, which can then be taken into account in the definition of the corresponding domains. The measurements and the constraints associated with the $2 \bar{k}$ equations given by (1) will be used to reduce the domains and thus the uncertainty on the variables, which can be formulated as a generalized set estimation problem.

The basic step of this generalized set estimation problem is to find the smallest domains $\widehat{\mathbb{P}}, \widehat{\mathbb{X}}_{0}$, $\widehat{\mathbb{W}}_{0}, \widehat{\mathbb{U}}_{0}, \widehat{\mathbb{X}}_{1}, \widehat{\mathbb{W}}_{1}, \widehat{\mathbb{U}}_{1}, \widehat{\mathbb{Y}}_{1}, \ldots, \widehat{\mathbb{X}}_{\bar{k}}, \widehat{\mathbb{W}}_{\bar{k}}, \widehat{\mathbb{U}}_{\bar{k}}$ and $\widehat{\mathbb{Y}}_{\bar{k}}$ such that the following implication is satisfied

$$
\left\{\begin{array} { l } 
{ ( 1 ) \text { hold true and } } \\
{ \mathbf { p } \in \mathbb { P } } \\
{ \mathbf { x } _ { 0 } \in \mathbb { X } _ { 0 } , \ldots , \mathbf { x } _ { \overline { k } } \in \mathbb { X } _ { \overline { k } } } \\
{ \mathbf { w } _ { 0 } \in \mathbb { W } _ { 0 } , \ldots , \mathbf { w } _ { \overline { k } } \in \mathbb { W } _ { \overline { k } } } \\
{ \mathbf { u } _ { 0 } \in \mathbb { U } _ { 0 } , \ldots , \mathbf { u } _ { \overline { k } } \in \mathbb { U } _ { \overline { k } } } \\
{ \mathbf { y } _ { 1 } \in \mathbb { Y } _ { 1 } , \ldots , \mathbf { y } _ { \overline { k } } \in \mathbb { Y } _ { \overline { k } } }
\end{array} \Rightarrow \left\{\begin{array}{l}
\mathbf{p} \in \widehat{\mathbb{P}} \\
\mathbf{x}_{0} \in \widehat{\mathbb{X}}_{0}, \ldots, \mathbf{x}_{\bar{k}} \in \widehat{\mathbb{X}}_{\bar{k}} \\
\mathbf{w}_{0} \in \widehat{\mathbb{W}}_{0}, \ldots, \mathbf{w}_{\bar{k}} \in \widehat{\mathbb{W}}_{\bar{k}} \\
\mathbf{u}_{0} \in \widehat{\mathbb{U}}_{0}, \ldots, \mathbf{u}_{\bar{k}} \in \widehat{\mathbb{U}}_{\bar{k}} \\
\mathbf{y}_{1} \in \widehat{\mathbb{Y}}_{1}, \ldots, \mathbf{y}_{\bar{k}} \in \widehat{\mathbb{Y}}_{\bar{k}}
\end{array}\right.\right.
$$


Since $\widehat{\mathbb{V}} \subset \mathbb{V}, \widehat{\mathbb{V}}$ can now replace $\mathbb{V}$ as a more accurate domain for $\mathbf{v}$. The operation thus performed can be written concisely as the instruction

$$
\mathcal{D}:=\mathcal{E}(\mathcal{D})
$$

The generalized set estimator based on (5) can then be sketched as follows:

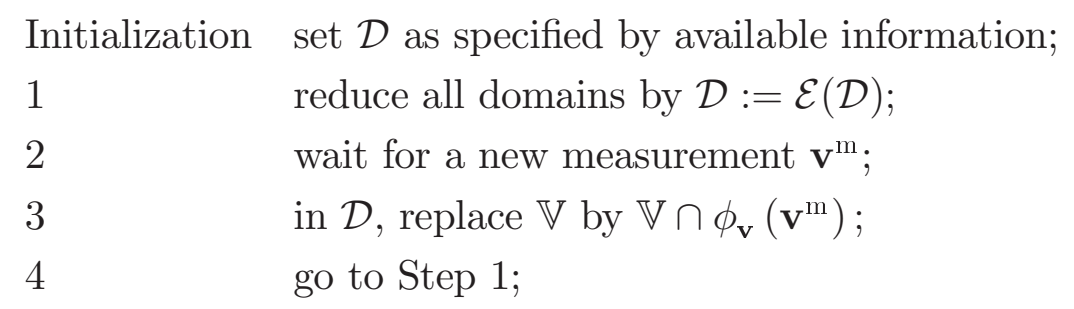

Note that in a noncausal context, all measurements are given at the initialization. Therefore, the estimation process stops after the first execution of Step 1.

Set parameter estimation, parameter tracking, state estimation and joint state and parameter estimation can all be seen as special cases of generalized set estimation. Moreover, the problem to be solved at Step 1 is itself a special case of a Set Constraint Satisfaction Problem (SCSP), to be presented in Section 2 in a more general context. In Section 3, constraint propagation techniques will be used to derive new set algorithms able to solve a large class of SCSPs, which includes the problem of Step 1. This general approach will be applied in Section 4 to causal and noncausal state estimation, and Section 5 will present an illustrative example.

\section{Set Constraint Satisfaction Problems}

This section presents some basic definitions and algorithms that are rather classical in the area of constraint propagation [3], [2], [5], but here these definitions and algorithms are extended to the case where intervals are replaced by more general subsets of real vector spaces.

Let $\mathcal{V}=\left\{\mathbf{v}_{1}, \ldots, \mathbf{v}_{n}\right\}$ be a finite set of vector variables with dimensions $d_{1} \in \mathbb{N}, \ldots, d_{n} \in \mathbb{N}$ and domains $\mathbb{V}_{1} \subset \mathbb{R}^{d_{1}}, \ldots, \mathbb{V}_{n} \subset \mathbb{R}^{d_{n}}$. The global space is the set $\mathbb{R}^{d}=\mathbb{R}^{d_{1}} \times \cdots \times \mathbb{R}^{d_{n}}$, where $d=d_{1}+\cdots+d_{n}$. The global domain is the set $\mathbb{V}=\mathbb{V}_{1} \times \cdots \times \mathbb{V}_{n}$. Of course, $\mathbb{V} \subset \mathbb{R}^{d}$. The subscript $i$ of a variable $\mathbf{v}_{i}$ and its domain $\mathbb{V}_{i}$ will be called their index.

Let $\mathbf{v}_{i}$ and $\mathbf{v}_{j}$ be two elements of $\mathcal{V}$. A binary constraint $\mathbb{C}_{i, j}$ over $\mathbf{v}_{i}$ and $\mathbf{v}_{j}$ is a subset of $\mathbb{R}^{d_{i}} \times \mathbb{R}^{d_{j}}$. Often this constraint can be put in the form

$$
\mathbb{C}_{i, j}=\left\{\left(\tilde{\mathbf{v}}_{i}, \tilde{\mathbf{v}}_{j}\right) \in \mathbb{R}^{d_{i}} \times \mathbb{R}^{d_{j}} \mid \tilde{\mathbf{v}}_{j}=\mathbf{f}_{j}\left(\tilde{\mathbf{v}}_{i}\right)\right\} \text {. }
$$


We shall then refer to it as $\mathbb{C}_{i, j}: \mathbf{v}_{j}=\mathbf{f}_{j}\left(\mathbf{v}_{i}\right)$. In this paper, we shall only consider binary constraints. Note that $n$-ary constraints with $n>2$ can always be decomposed into a set of binary constraints, so this is not limitative.

A Set Constraint Satisfaction Problem (SCSP) is a 3-uple $\mathcal{H}=(\mathcal{V}, \mathcal{D}, \mathcal{C})$, where $\mathcal{V}=\left\{\mathbf{v}_{1}, \ldots, \mathbf{v}_{n}\right\}$ is a finite set of variables, $\mathcal{D}=\left\{\mathbb{V}_{1}, \ldots, \mathbb{V}_{n}\right\}$ is the set of their domains and $\mathcal{C}$ is a finite set of binary constraints relating variables of $\mathcal{V}$.

Example 1 Consider three real numbers $x, y$ and $z$ related by the constraint $z=x^{2}+y^{2}$. Assume that $z$ is known to belong to the interval $[-3,1]$. This situation can be represented by an $\operatorname{SCSP} \mathcal{H}=(\mathcal{V}, \mathcal{D}, \mathcal{C})$, with $\mathcal{V}=\left\{\mathbf{v}_{1}, v_{2}\right\}, \mathcal{D}=\left\{\mathbb{V}_{1}, \mathbb{V}_{2}\right\}, \mathcal{C}=\left\{\mathbb{C}_{1,2}\right\}, \mathbf{v}_{1}=\left(\begin{array}{ll}x & )^{T}, v_{2}=\end{array}\right.$ $z, \mathbb{V}_{1}=\mathbb{R}^{2}, \mathbb{V}_{2}=[-3,1]$ and $\mathbb{C}_{1,2}: z=x^{2}+y^{2}$.

A point solution of $\mathcal{H}$ is a $n$-uple $\left(\tilde{\mathbf{v}}_{1}, \ldots, \tilde{\mathbf{v}}_{n}\right) \in \mathbb{V} \subset \mathbb{R}^{d}$ such that for all constraints $\mathbb{C}_{i, j} \in \mathcal{C}$, the pair $\left(\tilde{\mathbf{v}}_{i}, \tilde{\mathbf{v}}_{j}\right) \in \mathbb{C}_{i, j}$. The set of all point solutions of $\mathcal{H}$ is denoted by $\mathbb{S}(\mathcal{H})$. This set will be called the global solution set. In Example $1, \mathbb{S}=\left\{(x, y, z) \mid z=x^{2}+y^{2}\right.$ and $\left.z \in[-3,1]\right\}$, which is a cylinder with radius 1 .

The variable $\mathbf{v}_{i}$ is consistent in $\mathcal{H}$ (or $\mathcal{H}$-consistent) if

$$
\forall \tilde{\mathbf{v}}_{i} \in \mathbb{V}_{i}, \exists\left(\tilde{\mathbf{v}}_{1} \in \mathbb{V}_{1}, \ldots, \tilde{\mathbf{v}}_{i-1} \in \mathbb{V}_{i-1}, \tilde{\mathbf{v}}_{i+1} \in \mathbb{V}_{i+1}, \ldots, \tilde{\mathbf{v}}_{n} \in \mathbb{V}_{n}\right) \mid\left(\tilde{\mathbf{v}}_{1}, \ldots, \tilde{\mathbf{v}}_{n}\right) \in \mathbb{S}(\mathcal{H})
$$

In Example 1, neither $\mathbf{v}_{1}$ nor $v_{2}$ is $\mathcal{H}$-consistent. If the domain $\mathbb{V}_{1}$ is replaced by the disk centered at 0 and with a radius equal to 0.5 , denoted by $\operatorname{Disk}(0,0.5)$, then $\mathbf{v}_{1}$ becomes $\mathcal{H}$ consistent. If $\mathbb{V}_{2}$ is replaced by the interval $[0,0.25]$, then $v_{2}$ becomes $\mathcal{H}$-consistent. Note that if $\mathbf{v}_{i}$ is $\mathcal{H}$-consistent and if $\mathbb{V}_{i}$ is replaced by any subset of $\mathbb{V}_{i}$, then $\mathbf{v}_{i}$ is still consistent in the new SCSP.

If $\mathcal{I}=\left\{i_{1}, \ldots, i_{p}\right\}$ is a subset of the set of indices $\{1, \ldots, n\}, \mathcal{H}^{\prime}=\left(\mathcal{V}^{\prime}, \mathcal{D}^{\prime}, \mathcal{C}^{\prime}\right)$, where $\mathcal{V}^{\prime} \triangleq$ $\left\{\mathbf{v}_{i_{1}}, \ldots, \mathbf{v}_{i_{p}}\right\}, \mathcal{D}^{\prime} \triangleq\left\{\mathbb{V}_{i_{1}}, \ldots, \mathbb{V}_{i_{p}}\right\}$ and $\mathcal{C}^{\prime} \triangleq\left\{\mathbb{C}_{i, j} \in \mathcal{C}\right.$ such that $i \in \mathcal{I}$ and $\left.j \in \mathcal{I}\right\}$, is called a subSCSP of $\mathcal{H}$. It is trivial to show that if $\mathbf{v}_{i} \in \mathcal{V}^{\prime}$ is $\mathcal{H}$-consistent, it is also $\mathcal{H}^{\prime}$-consistent.

The $i$ th projected domain $\mathbb{S}_{i}=\pi_{i}(\mathcal{H})$ onto the variable $\mathbf{v}_{i}$ is the largest domain $\mathbb{S}_{i} \subset \mathbb{V}_{i}$ such that if we replace $\mathbb{V}_{i}$ by $\mathbb{S}_{i}$ in $\mathcal{H}, \mathbf{v}_{i}$ becomes $\mathcal{H}$-consistent. It can also be defined by the orthogonal projection of the global solution set $\mathbb{S}(\mathcal{H})$ onto $\mathbb{R}^{d_{i}}$, i.e.,

$$
\begin{aligned}
& \tilde{\mathbf{v}}_{i} \in \pi_{i}(\mathcal{H}) \\
\Leftrightarrow & \exists\left(\tilde{\mathbf{v}}_{1} \in \mathbb{V}_{1}, \ldots, \tilde{\mathbf{v}}_{i-1} \in \mathbb{V}_{i-1}, \tilde{\mathbf{v}}_{i+1} \in \mathbb{V}_{i+1}, \ldots, \tilde{\mathbf{v}}_{n} \in \mathbb{V}_{n}\right) \mid\left(\tilde{\mathbf{v}}_{1}, \ldots, \tilde{\mathbf{v}}_{n}\right) \in \mathbb{S}(\mathcal{H}) .
\end{aligned}
$$

Note that if $\mathbf{v}_{i}$ is $\mathcal{H}$-consistent, $\mathbb{V}_{i}=\pi_{i}(\mathcal{H})$. In Example $1, \pi_{1}(\mathcal{H})=\operatorname{Disk}(0,1)$ and $\pi_{2}(\mathcal{H})=$ $[0,1]$. 
$\mathcal{H}$ is an elementary $S C S P$ if $\mathcal{V}$ is a singleton $\left\{\mathbf{v}_{1}\right\}$. Therefore, $\mathcal{C}=\emptyset$ and $\mathbb{V}_{1}$ is necessarily $\mathcal{H}$-consistent.

Two SCSPs $\mathcal{H}=(\mathcal{V}, \mathcal{D}, \mathcal{C})$ and $\mathcal{H}^{\prime}=\left(\mathcal{V}^{\prime}, \mathcal{D}^{\prime}, \mathcal{C}^{\prime}\right)$ are equivalent, denoted by $\mathcal{H} \equiv \mathcal{H}^{\prime}$, if $\mathcal{V}=$ $\mathcal{V}^{\prime}, \mathcal{C}=\mathcal{C}^{\prime}$ and $\mathbb{S}(\mathcal{H})=\mathbb{S}\left(\mathcal{H}^{\prime}\right)$. We shall say that $\mathcal{H}$ is a contraction of $\mathcal{H}^{\prime}$ if $\mathcal{H} \equiv \mathcal{H}^{\prime}$ and $\mathbb{V}_{i} \subset \mathbb{V}_{i}^{\prime}$ for any index $i$. To contract an SCSP is to replace it by one of its contractions. $\mathcal{H}$ is minimal if it admits no contraction of itself but itself.

$\mathcal{H}^{\prime}$ is the optimal contraction of $\mathcal{H}$ if $\mathcal{H} \equiv \mathcal{H}^{\prime}$ and $\mathcal{H}^{\prime}$ is minimal. We shall write $\mathcal{H}^{\prime}=\mathcal{E}(\mathcal{H})$ or sometimes $\mathcal{D}^{\prime}=\mathcal{E}(\mathcal{D})$ as in $(5)$, since $\mathcal{H}$ and $\mathcal{H}^{\prime}$ can only differ by their domains. The $n$ domains $\mathbb{V}_{i}^{\prime}$ of the optimal contraction satisfy $\mathbb{V}_{i}^{\prime}=\pi_{i}(\mathcal{H}), i \in\{1, \ldots, n\}$.

Consider two variables $\mathbf{v}_{i}$ and $\mathbf{v}_{j}$ related by a constraint $\mathbb{C}_{i, j}$. The local contraction operator of the domain $\mathbb{V}_{i}$ with respect to the variable $\mathbf{v}_{j}$ is defined as

$$
\rho_{j}\left(\mathbb{V}_{i}\right)=\left\{\tilde{\mathbf{v}}_{i} \in \mathbb{V}_{i} \mid \exists \tilde{\mathbf{v}}_{j} \in \mathbb{V}_{j},\left(\tilde{\mathbf{v}}_{i}, \tilde{\mathbf{v}}_{j}\right) \in \mathbb{C}_{i, j}\right\} .
$$

Note that $\rho_{j}\left(\mathbb{V}_{i}\right) \subset \mathbb{V}_{i}$. The new SCSP obtained by replacing $\mathbb{V}_{i}$ by $\mathbb{V}_{i}^{\prime}=\rho_{j}\left(\mathbb{V}_{i}\right)$ is thus a contraction of the former SCSP. Figure 1 illustrates this definition. If the constraint $\mathbb{C}_{i, j}$ is given by $\mathbb{C}_{i, j}: \mathbf{v}_{j}=\mathbf{f}_{j}\left(\mathbf{v}_{i}\right)$, then

$$
\begin{aligned}
\rho_{j}\left(\mathbb{V}_{i}\right) & =\mathbb{V}_{i} \cap \mathbf{f}_{j}^{-1}\left(\mathbb{V}_{j}\right), \\
\rho_{i}\left(\mathbb{V}_{j}\right) & =\mathbb{V}_{j} \cap \mathbf{f}_{j}\left(\mathbb{V}_{i}\right) .
\end{aligned}
$$

In Example 1, if $f(x, y)=x^{2}+y^{2}$, then

$$
\begin{aligned}
& \rho_{1}\left(\mathbb{V}_{2}\right)=[-3,1] \cap f\left(\mathbb{R}^{2}\right)=[-3,1] \cap[0, \infty[=[0,1], \\
& \rho_{2}\left(\mathbb{V}_{1}\right)=\mathbb{R}^{2} \cap f^{-1}([-3,1])=\operatorname{Disk}(0,1) .
\end{aligned}
$$

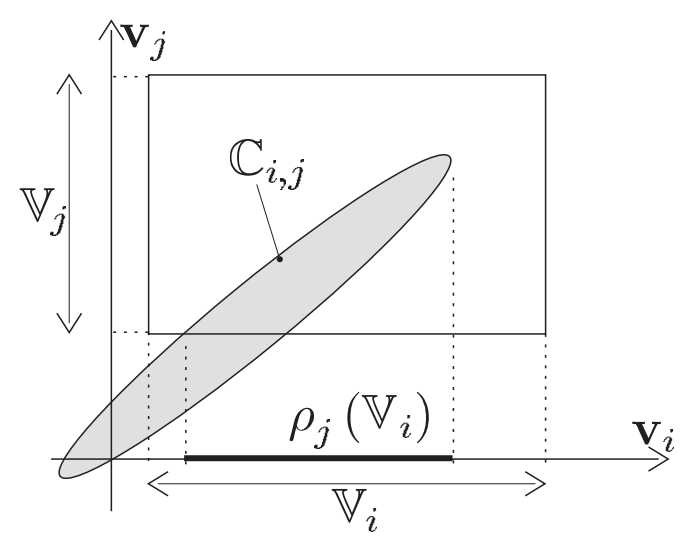

Figure 1: Local contraction operator 
The Waltz algorithm [22], [3] is one of the basic algorithms that can be used to perform contractions of SCSPs. Its principle is to choose any constraint in $\mathcal{C}$ and to contract the domains of the two associated variables $\mathbf{v}_{i}$ and $\mathbf{v}_{j}$ using the local contraction operators $\rho_{i}$ and $\rho_{j}$. This is continued until no constraint in $\mathcal{C}$ is able to contract any domain. Unfortunately, the resulting SCSP may be non-minimal, because the algorithm may come to a deadlock, as illustrated by the following example.

Example 2 Consider the $S C S P \mathcal{H}=(\mathcal{V}, \mathcal{D}, \mathcal{C})$, where

$$
\begin{aligned}
& \mathcal{V}=\left\{v_{1}, v_{2}, v_{3}\right\} \\
& \mathcal{D}=\left\{\mathbb{V}_{1}=[-1,1] ; \mathbb{V}_{2}=[-1,1] ; \mathbb{V}_{3}=[-1,1]\right\} \\
& \mathcal{C}=\left\{\mathbb{C}_{1,2}: v_{2}=-v_{1} ; \mathbb{C}_{2,3}: v_{3}=-v_{2} ; \mathbb{C}_{3,1}: v_{1}=-v_{3}\right\}
\end{aligned}
$$

Although the only solution is $v_{1}=v_{2}=v_{3}=0$, the Waltz algorithm is unable to contract the domains $\mathbb{V}_{i}$.

The graph of any given SCSP $\mathcal{H}$ can be constructed as follows. To each variable $\mathbf{v}_{i}$, is associated a node and to each binary constraint $\mathbb{C}_{i, j}$ is associated an arc between the nodes $\mathbf{v}_{i}$ et $\mathbf{v}_{j}$. The graph associated with Example 2 is depicted on Figure 2. The failure of the Waltz algorithm to contract this SCSP is due to the fact that its graph contains a cycle.

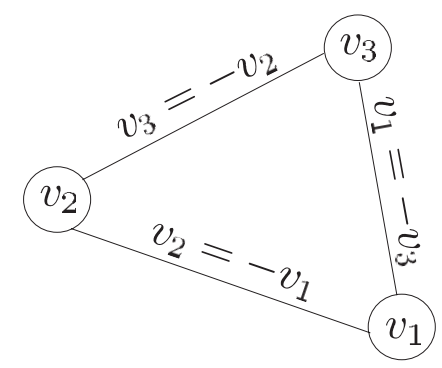

Figure 2: Graph of a SCSP with a cycle

When the graph is a tree, i.e., a connected graph without cycles, the Waltz algorithm converges to the optimal contraction of $\mathcal{H}$. This result is a direct consequence of Theorem 6 in Section 3 , which corresponds to a more efficient contraction algorithm.

\section{Contraction algorithms}

When the graph of the SCSP is a tree, the Waltz algorithm produces an optimal contraction, but not very efficiently because the constraints are taken into account in arbitrary order. In 
this section, we propose a new algorithm to contract an SCSP whose graph is a tree, which also produces an optimal contraction, but in a much more efficient way. It extends to more general domains the propagation-retropropagation algorithm proposed in [1], and is based on the next two theorems.

\subsection{Propagation and retropropagation theorems}

Theorem 1 (propagation theorem): Let $\mathcal{H}_{a}=\left(\mathcal{V}_{a}, \mathcal{D}_{a}, \mathcal{C}_{a}\right)$ and $\mathcal{H}_{b}=\left(\mathcal{V}_{b}, \mathcal{D}_{b}, \mathcal{C}_{b}\right)$ be two SCSPs with all their variables distinct. Let $\mathbf{v}_{a 1} \in \mathcal{V}_{a}$ and $\mathbf{v}_{b 1} \in \mathcal{V}_{b}$ be two variables related by the constraint $\mathbb{C}_{a 1, b 1}$. The new $S C S P \mathcal{H}=(\mathcal{V}, \mathcal{D}, \mathcal{C})$, where

$$
\mathcal{V}=\mathcal{V}_{a} \cup \mathcal{V}_{b} ; \mathcal{D}=\mathcal{D}_{a} \cup \mathcal{D}_{b} ; \mathcal{C}=\mathcal{C}_{a} \cup \mathcal{C}_{b} \cup\left\{\mathbb{C}_{a 1, b 1}\right\}
$$

is such that if $\mathbf{v}_{a 1}$ is $\mathcal{H}_{a}$-consistent and $\mathbf{v}_{b 1}$ is $\mathcal{H}_{b}$-consistent, then $\pi_{a 1}(\mathcal{V}, \mathcal{D}, \mathcal{C})=\rho_{b 1}\left(\mathbb{V}_{a 1}\right)$ and $\pi_{b 1}(\mathcal{V}, \mathcal{D}, \mathcal{C})=\rho_{a 1}\left(\mathbb{V}_{b 1}\right)$.

Proof: Because of the symmetry of the problem, it suffices to prove that $\pi_{a 1}(\mathcal{H})=\rho_{b 1}\left(\mathbb{V}_{a 1}\right)$. Let $\mathbf{v}_{a 2}, \ldots, \mathbf{v}_{a p}$ be the $p-1$ variables of $\mathcal{V}_{a}$ distinct from $\mathbf{v}_{a 1}$ and $\mathbf{v}_{b 2}, \ldots, \mathbf{v}_{b q}$ be the $q-1$ variables of $\mathcal{V}_{b}$ distinct from $\mathbf{v}_{b 1}$. Since $\mathbf{v}_{a 1}$ is $\mathcal{H}_{a}$-consistent and $\mathbf{v}_{b 1}$ is $\mathcal{H}_{b}$-consistent, $\pi_{a 1}\left(\mathcal{H}_{a}\right)=\mathbb{V}_{a 1}$ and $\pi_{b 1}\left(\mathcal{H}_{b}\right)=\mathbb{V}_{b 1}$. Now, $\tilde{\mathbf{v}}_{a 1} \in \pi_{a 1}(\mathcal{H}) \Leftrightarrow \tilde{\mathbf{v}}_{a 1} \in \mathbb{V}_{a 1}$ and $\exists \tilde{\mathbf{v}}_{a 2} \in \mathbb{V}_{a 2}, \ldots, \exists \tilde{\mathbf{v}}_{a p} \in \mathbb{V}_{a p}, \exists \tilde{\mathbf{v}}_{b 1} \in \mathbb{V}_{b 1}$, $\ldots, \exists \tilde{\mathbf{v}}_{b n} \in \mathbb{V}_{b n}$ such that all constraints of $\mathcal{C}$ are satisfied, i.e.,

$$
\begin{aligned}
& \tilde{\mathbf{v}}_{a 1} \in \pi_{a 1}(\mathcal{H}) \\
& \Leftrightarrow\left\{\begin{array}{l}
\left(\text { i) } \exists \tilde{\mathbf{v}}_{a 2} \in \mathbb{V}_{a 2}, \ldots, \exists \tilde{\mathbf{v}}_{a p} \in \mathbb{V}_{a p} \mid\left(\tilde{\mathbf{v}}_{a 1}, \ldots, \tilde{\mathbf{v}}_{a p}\right) \in \mathbb{S}\left(\mathcal{H}_{a}\right)\right. \\
\text { (ii) } \exists \tilde{\mathbf{v}}_{b 1} \in \mathbb{V}_{b 1}, \ldots, \exists \tilde{\mathbf{v}}_{b n} \in \mathbb{V}_{b n} \mid\left\{\begin{array}{c}
\left(\tilde{\mathbf{v}}_{a 1}, \tilde{\mathbf{v}}_{b 1}\right) \in \mathbb{C}_{a 1, b 1} \\
\left(\tilde{\mathbf{v}}_{b 1}, \ldots, \tilde{\mathbf{v}}_{b q}\right) \in \mathbb{S}\left(\mathcal{H}_{b}\right)
\end{array}\right.
\end{array}\right.
\end{aligned}
$$

Since $\mathbb{V}_{a 1}=\pi_{a 1}\left(\mathcal{H}_{a}\right)$, (i) is equivalent to $\tilde{\mathbf{v}}_{a 1} \in \mathbb{V}_{a 1}$. Since $\mathbb{V}_{b 1}=\pi_{b 1}\left(\mathcal{H}_{b}\right)$, (ii) is equivalent to $\exists \tilde{\mathbf{v}}_{b 1} \in \mathbb{V}_{b 1} \mid\left(\tilde{\mathbf{v}}_{a 1}, \tilde{\mathbf{v}}_{b 1}\right) \in \mathbb{C}_{a 1, b 1}$. The equivalence (13) becomes

$$
\tilde{\mathbf{v}}_{a 1} \in \pi_{a 1}(\mathcal{H}) \Leftrightarrow\left\{\begin{array}{l}
\tilde{\mathbf{v}}_{a 1} \in \mathbb{V}_{a 1} \\
\exists \tilde{\mathbf{v}}_{b 1} \in \mathbb{V}_{b 1} \mid\left(\tilde{\mathbf{v}}_{a 1}, \tilde{\mathbf{v}}_{b 1}\right) \in \mathbb{C}_{a 1, b 1}
\end{array}\right\} \Leftrightarrow \tilde{\mathbf{v}}_{a 1} \in \rho_{b 1}\left(\mathbb{V}_{a 1}\right) .
$$

Figure 3 illustrates how the two former SCSPs $\mathcal{H}_{a}$ and $\mathcal{H}_{b}$ related by the constraint $\mathbb{C}_{a 1, b 1}$ form the new SCSP $\mathcal{H}$.

Corollary 2 Let $\mathcal{H}_{b}=\left(\mathcal{V}_{b}, \mathcal{D}_{b}, \mathcal{C}_{b}\right)$ be an SCSP and $\mathbf{v}_{a}$ be a new variable with domain $\mathbb{V}_{a}$ related to a variable $\mathbf{v}_{b 1} \in \mathcal{V}_{b}$ by the constraint $\mathbb{C}_{a, b 1}$. In the $\operatorname{SCSP\mathcal {H}}=(\mathcal{V}, \mathcal{D}, \mathcal{C})$, where

$$
\mathcal{V}=\mathcal{V}_{b} \cup\left\{\mathbf{v}_{a}\right\} ; \mathcal{D}=\mathcal{D}_{b} \cup\left\{\mathbb{V}_{a}\right\} ; \mathcal{C}=\mathcal{C}_{b} \cup\left\{\mathbb{C}_{a, b 1}\right\},
$$

if $\mathbf{v}_{b 1}$ is $\mathcal{H}_{b}$-consistent then $\pi_{a}(\mathcal{H})=\rho_{b 1}\left(\mathbb{V}_{a}\right)$. 


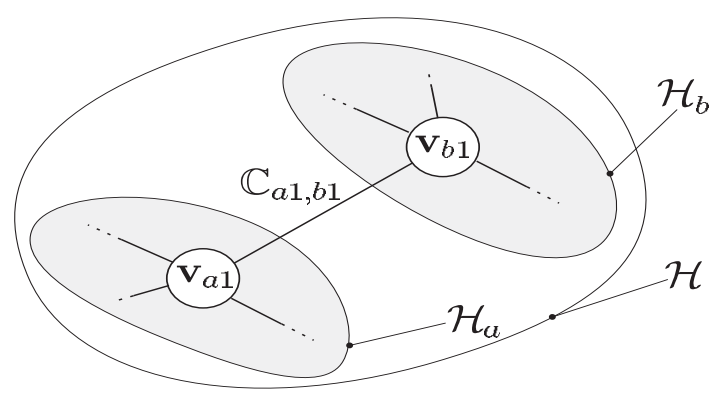

Figure 3: The three SCSPs involved in the propagation theorem

Proof: Consider the elementary SCSP $\mathcal{H}_{a}=\left(\mathcal{V}_{a}, \mathcal{D}_{a}, \mathcal{C}_{a}\right)$ where $\mathcal{V}_{a}=\left\{\mathbf{v}_{a}\right\}, \mathcal{D}_{a}=\left\{\mathbb{V}_{a}\right\}$ and $\mathcal{C}_{a}=\emptyset$. Theorem 1 implies that $\pi_{a}(\mathcal{H})=\rho_{b 1}\left(\mathbb{V}_{a}\right)$.

Theorem 3 (retropropagation theorem): Let $\mathcal{H}_{a}=\left(\mathcal{V}_{a}, \mathcal{D}_{a}, \mathcal{C}_{a}\right)$ and $\mathcal{H}_{b}=\left(\mathcal{V}_{b}, \mathcal{D}_{b}, \mathcal{C}_{b}\right)$ be two SCSPs with all their variables distinct. Let $\mathbf{v}_{a 1} \in \mathcal{V}_{a}$ and $\mathbf{v}_{b 1} \in \mathcal{V}_{b}$ be two variables related by the constraint $\mathbb{C}_{a 1, b 1}$. Consider the $S C S P \mathcal{H}=(\mathcal{V}, \mathcal{D}, \mathcal{C})$ :

$$
\mathcal{V}=\mathcal{V}_{a} \cup \mathcal{V}_{b} ; \mathcal{D}=\mathcal{D}_{a} \cup \mathcal{D}_{b} ; \mathcal{C}=\mathcal{C}_{a} \cup \mathcal{C}_{b} \cup\left\{\mathbb{C}_{a 1, b 1}\right\}
$$

If $\mathbf{v}_{a 1}$ is $\mathcal{H}$-consistent and $\mathbf{v}_{b 1}$ is $\mathcal{H}_{b}$-consistent, then

$$
\pi_{b 1}(\mathcal{H})=\rho_{a 1}\left(\mathbb{V}_{b 1}\right)
$$

Proof: Since $\mathcal{H}_{a}$ is a subSCSP of $\mathcal{H}$ and since $\mathbf{v}_{a 1}$ is $\mathcal{H}$-consistent, $\mathbf{v}_{a 1}$ is also $\mathcal{H}_{a}$-consistent. From Theorem $1, \pi_{b 1}(\mathcal{V}, \mathcal{D}, \mathcal{C})=\rho_{a 1}\left(\mathbb{V}_{b 1}\right)$.

\subsection{Algorithms FALL and CLIMB}

This section describes two efficient contraction algorithms that can be used to contract optimally an SCSP $\mathcal{H}$, when its graph is a tree. FALL, based on the propagation theorem, scans the tree from its leaves down to its root and CLIMB, based on the retropropagation theorem, scans it from its root up to its leaves. It will be shown that a single execution of FALL followed by a single execution of CLIMB leads to the optimal contraction of $\mathcal{H}$.

Remark 1 Any SCSP containing cycles can be transformed into an equivalent SCSP whose graph is a tree. It suffices for that to group all variables responsible for the existence of a cycle into a single vector variable. 
To present these two algorithms, some notations concerning trees will be needed.

\subsubsection{Trees}

The trees to be considered are not directed. A rooted tree is obtained after selecting any node of a given tree $\mathcal{T}$ as its root. By analogy, with the forest variety, we shall say that the root is the lowest node of the tree. A node $\mathbf{v}_{a}$ is over a node $\mathbf{v}_{b}$ (or equivalently $\mathbf{v}_{b}$ is below $\mathbf{v}_{a}$ ) if the subtree $\mathcal{T}_{a}$ of $\mathcal{T}$ with root $\mathbf{v}_{a}$ contains $\mathbf{v}_{b}$. Consider a tree $\mathcal{T}$, with root $\mathbf{v}_{1}$. By removing $\mathbf{v}_{1}$, one obtains $q$ subtrees $\mathcal{T}_{i_{1}}, \ldots, \mathcal{T}_{i_{q}}$ of $\mathcal{T}$. These $q$ subtrees are the sons of $\mathcal{T}$. A tree with no son is a leaf. If $\mathcal{T}$ is a leaf, we shall write $\mathcal{T}=\mathbf{v}_{1}$. Otherwise, we shall write $\mathcal{T}=\mathbf{v}_{1} \rightarrow\left(\mathcal{T}_{i_{1}}|\ldots| \mathcal{T}_{i_{q}}\right)$. Each son of $\mathcal{T}$ will be rooted in a natural way by choosing its node that is connected to $\mathbf{v}_{1}$ as its root.

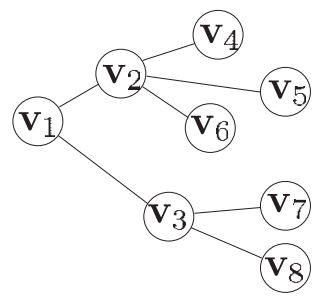

Figure 4: Example of a tree with eight nodes

Example 3 When $\mathbf{v}_{1}$ is chosen as its root, the tree $\mathcal{T}$ of Figure 4 will be denoted by

$$
\mathcal{T}=\mathbf{v}_{1} \rightarrow\left(\mathbf{v}_{2} \rightarrow\left(\mathbf{v}_{4}\left|\mathbf{v}_{5}\right| \mathbf{v}_{6}\right) \mid \mathbf{v}_{3} \rightarrow\left(\mathbf{v}_{7} \mid \mathbf{v}_{8}\right)\right)
$$

Its two sons are $\mathcal{T}_{2}=\mathbf{v}_{2} \rightarrow\left(\mathbf{v}_{4}\left|\mathbf{v}_{5}\right| \mathbf{v}_{6}\right)$ and $\mathcal{T}_{3}=\mathbf{v}_{3} \rightarrow\left(\mathbf{v}_{7} \mid \mathbf{v}_{8}\right)$. $\mathcal{T}$ has seven subtrees, five of which are leaves.

A tree $S C S P$ (or TSCSP) $\mathcal{H}$ is an SCSP, the graph of which is a tree. A TSCSP can be rooted by selecting one of its node $\mathbf{v}_{i}$ as its root. Only rooted TSCSPs will be considered from now on, and we shall write $\mathcal{H}=\left(\mathcal{V}, \mathcal{D}, \mathcal{C}, \mathbf{v}_{i}\right)$ to denote a TSCSP with root $\mathbf{v}_{i}$.. All notions (root, leaves, sons...) existing for trees extend to TSCSPs in a natural way.

Let $\mathcal{H}=\left(\mathcal{V}, \mathcal{D}, \mathcal{C}, \mathbf{v}_{i}\right)$ be a TSCSP and $\mathbf{v}_{j}$ be an element of $\mathcal{V}$. Let $\mathcal{H}_{j}=\operatorname{SubTree}\left(\mathcal{H}, \mathbf{v}_{j}\right)$ be the subTSCSP of $\mathcal{H}$ with root $\mathbf{v}_{j}$ associated with all nodes that are over $\mathbf{v}_{j}$. A variable $\mathbf{v}_{j}$ is up-consistent in $\mathcal{H}$ if it is consistent in $\mathcal{H}_{j}=\operatorname{Subtree}\left(\mathcal{H}, \mathbf{v}_{j}\right) . \mathcal{H}$ is up-consistent if all of its nodes are up-consistent in $\mathcal{H}$. 


\subsubsection{Algorithm FALL}

An up-consistent contraction of any $\operatorname{TSCSP} \mathcal{H}=\left(\mathcal{V}, \mathcal{D}, \mathcal{C}, \mathbf{v}_{i}\right)$ can be computed by the following recursive algorithm.

\section{FALL(inout: $\mathcal{H})$}

1 if $\mathcal{H}$ is a leaf return;

$2 i:=\operatorname{index}(\operatorname{root}(\mathcal{H})) ; q:=$ number of sons of $\mathcal{H}$;

3 for all sons $\mathcal{H}_{j_{k}}$ of $\mathcal{H}, k \in\{1, \ldots, q\}$

$4 \operatorname{FALL}\left(\mathcal{H}_{j_{k}}\right)$;

$5 \quad j_{k}:=\operatorname{index}\left(\operatorname{root}\left(\mathcal{H}_{j_{k}}\right)\right)$;

$6 \quad \mathbb{V}_{i}:=\rho_{j_{k}}\left(\mathbb{V}_{i}\right) ;$

7 endfor.

Theorem 4 : FALL generates an up-consistent contraction of any TSCSP.

Proof: The proof is in two parts. We shall first prove, by induction on $k$, that if $\operatorname{FALL}\left(\mathcal{H}_{j_{k}}\right)$ makes $\mathcal{H}_{j_{k}}$ up-consistent at Step 4 , then $\mathcal{H}$ is up-consistent after completion of FALL. Denote by $\mathcal{H}_{i}(k)$ be the subtree of $\mathcal{H}$, with the same root $\mathbf{v}_{i}$ and with $k$ sons $\mathcal{H}_{j_{1}}, \mathcal{H}_{j_{2}}, \ldots, \mathcal{H}_{j_{k}}$, in common with $\mathcal{H}, k \in\{1, \ldots, q\}$. For $k=1$, from Corollary $2, \mathcal{H}_{i}(1)$ is up-consistent after Step 6 (take $a=i$ and $b=j_{1}$ ). Assume now that $\mathcal{H}_{i}(k)$ is up-consistent. From Theorem 1 (take $\mathcal{H}_{a}$ for the SCSP associated with $\mathcal{H}_{i}(k)$ and $\left.b=j_{k+1}\right), \mathcal{H}_{i}(k+1)$ is up-consistent after the $(k+1)$ th execution of Step 6. Therefore $\mathcal{H}=\mathcal{H}_{i}(q)$ is up-consistent at Step 7. We shall now complete the proof by induction. If $\mathcal{H}$ is a leaf, it is already up-consistent and the theorem holds true. Assume that all sons $\mathcal{H}_{j_{1}}, \ldots, \mathcal{H}_{j_{q}}$ of $\mathcal{H}$ have been made up-consistent by FALL. From the first part of the proof, $\operatorname{FALL}(\mathcal{H})$ then makes $\mathcal{H}$ up-consistent.

Example 4 Consider the $T S C S P \mathcal{H}=\left(\mathcal{V}, \mathcal{D}, \mathcal{C}, \mathbf{v}_{1}\right)$ associated with the tree (18). FALL executes the following sequence of set operations

$$
\begin{aligned}
\mathbb{V}_{2}: & =\mathbb{V}_{2} \cap \rho_{4}\left(\mathbb{V}_{2}\right) \cap \rho_{5}\left(\mathbb{V}_{2}\right) \cap \rho_{6}\left(\mathbb{V}_{2}\right) \\
\mathbb{V}_{3} & :=\mathbb{V}_{3} \cap \rho_{7}\left(\mathbb{V}_{3}\right) \cap \rho_{8}\left(\mathbb{V}_{3}\right), \\
\mathbb{V}_{1}: & =\mathbb{V}_{1} \cap \rho_{2}\left(\mathbb{V}_{1}\right) \cap \rho_{3}\left(\mathbb{V}_{1}\right),
\end{aligned}
$$

which makes $\mathcal{H}$ up-consistent. The variable $\mathbf{v}_{1}$ is then also $\mathcal{H}$-consistent, i.e., $\mathbb{V}_{1}=\pi_{1}(\mathcal{H})$. 


\subsubsection{Algorithm CLIMB}

Let $\mathcal{H}=\left(\mathcal{V}, \mathcal{D}, \mathcal{C}, \mathbf{v}_{1}\right)$ be an up-consistent TSCSP. This consistency may result from an execution of $\operatorname{FALL}(\mathcal{H})$. We shall now give an algorithm to compute $\mathcal{E}(\mathcal{H})$, the minimal contraction of $\mathcal{H}$. The principle of CLIMB is to propagate the $\mathcal{H}$-consistency of $\mathbf{v}_{1}$, from the root up to the leaves. One step of Climb is now described. Consider an $\mathcal{H}$-consistent variable $\mathbf{v}_{i}$ and one of its sons $\mathbf{v}_{j}$. Cut the arc between the nodes $\mathbf{v}_{i}$ and $\mathbf{v}_{j}$ (see Figure 5). Two TSCSPs are thus generated. One of them is $\mathcal{H}_{j}=\left(\mathcal{V}_{j}, \mathcal{D}_{j}, \mathcal{C}_{j}, \mathbf{v}_{j}\right)=\operatorname{SubTree}\left(\mathcal{H}, \mathbf{v}_{j}\right)$. Since $\mathcal{H}$ is up-consistent, $\mathbf{v}_{j}$ is $\mathcal{H}_{j}$-consistent. From Theorem 3 (where $a 1=i, b 1=j$ ), $\pi_{j}(\mathcal{H})=\rho_{i}\left(\mathbb{V}_{j}\right)$. This reasoning can be applied to all nodes of $\mathcal{H}$, from its root to its leaves. The following recursive algorithm is thus obtained.

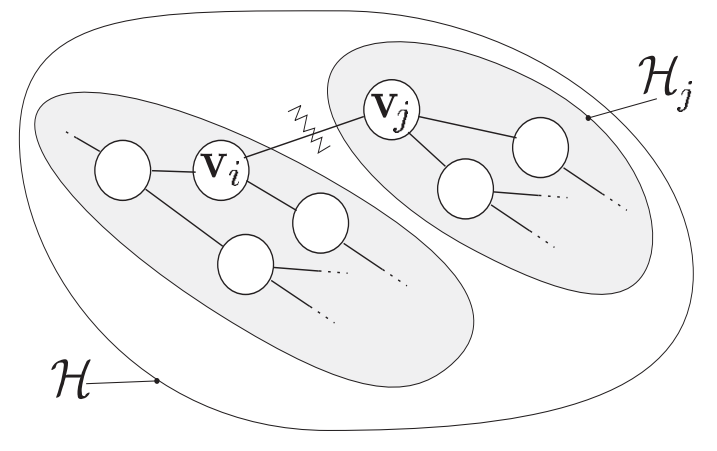

Figure 5: Illustration of the retropropagation step

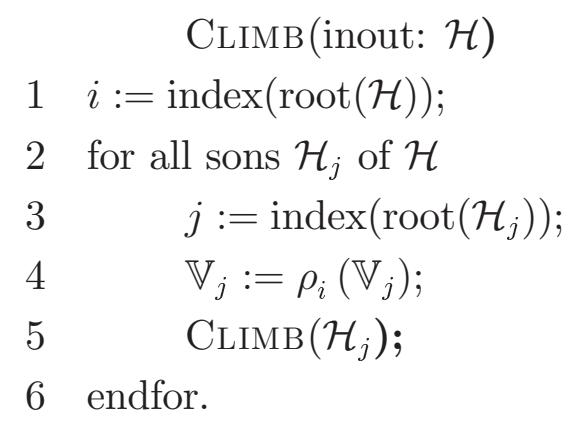

Theorem 5 ClimB computes the optimal contraction of any up-consistent TSCSP.

Proof: The proof is by induction. Since $\mathcal{H}=\left(\mathcal{V}, \mathcal{D}, \mathcal{C}, \mathbf{v}_{1}\right)$ is up-consistent, $\mathbf{v}_{1}$ is $\mathcal{H}$-consistent. Assume now that the root $\mathbf{v}_{i}$ of the current subtree $\mathcal{H}_{i}$, is $\mathcal{H}$-consistent. Consider a son $\mathbf{v}_{j}$ of $\mathbf{v}_{i}$. After Step 4, from Theorem 3 (where $a 1=i$ and $b 1=j$ ), $\mathbf{v}_{j}$ is $\mathcal{H}$-consistent. After completion of the algorithm, all variables are thus $\mathcal{H}$-consistent. 
Example 5 Consider again the TSCSP $\mathcal{H}$ associated with the tree given by (18). Assume that $\mathcal{H}$ has been made up-consistent by FALL as in Example 4. CLIMB generates the following sequence of set operations: $\mathbb{V}_{2}:=\mathbb{V}_{2} \cap \rho_{1}\left(\mathbb{V}_{2}\right) ; \mathbb{V}_{4}:=\mathbb{V}_{4} \cap \rho_{2}\left(\mathbb{V}_{4}\right) ; \mathbb{V}_{5}:=\mathbb{V}_{5} \cap \rho_{2}\left(\mathbb{V}_{5}\right)$; $\mathbb{V}_{6}:=\mathbb{V}_{6} \cap \rho_{2}\left(\mathbb{V}_{6}\right) ; \mathbb{V}_{3}:=\mathbb{V}_{3} \cap \rho_{1}\left(\mathbb{V}_{3}\right) ; \mathbb{V}_{7}:=\mathbb{V}_{7} \cap \rho_{3}\left(\mathbb{V}_{7}\right) ; \mathbb{V}_{8}:=\mathbb{V}_{8} \cap \rho_{3}\left(\mathbb{V}_{8}\right)$. From Theorem $5, \mathcal{H}$ is now minimal.

\title{
3.2.4 Algorithm FALL-CLIMB
}

FALL and Climb can be combined to compute the minimal contraction of a given TSCSP $\mathcal{H}$. The resulting algorithm is:

\author{
FALL-Climb(inout: $\mathcal{H}$ ) \\ $1 \operatorname{FALL}(\mathcal{H}) ;$ \\ $2 \operatorname{CLimb}(\mathcal{H})$.
}

Theorem 6 FALL-CLIMB returns the optimal contraction of any TSCSP.

Proof: Denote by $\mathcal{H}(0)$ the initial TSCSP, by $\mathcal{H}(1)$ the TSCSP after Step 1 and by $\mathcal{H}(2)$ the TSCSP after Step 2. From Theorem $4, \mathcal{H}(1)$ is up-consistent and equivalent to $\mathcal{H}(0)$. From Theorem $5, \mathcal{H}(2)$ is minimal and equivalent to $\mathcal{H}(0)$.

\section{Application to state estimation}

To facilitate understanding, we shall consider the autonomous discrete-time system:

$$
\left\{\begin{array}{l}
\mathbf{x}_{k}=\mathbf{f}_{k}\left(\mathbf{x}_{k-1}\right) \\
\mathbf{y}_{k}=\mathbf{g}_{k}\left(\mathbf{x}_{k}\right)
\end{array} \quad k=1, \ldots, \bar{k}\right.
$$

where $\mathbf{x}_{k} \in \mathbb{R}^{n}$ is the state vector and $\mathbf{y}_{k} \in \mathbb{R}^{m}$ is the output vector. It is a special case of the problem defined by (1), which could be treated in its general form along the same lines. The functions $\mathbf{f}_{k}$ and $\mathbf{g}_{k}$ may be nonlinear. At time $k$, the state estimator can use the measurement $\mathbf{y}_{1}^{\mathrm{m}}, \ldots, \mathbf{y}_{k}^{\mathrm{m}}$ in the causal case, and $\mathbf{y}_{1}^{\mathrm{m}}, \ldots, \mathbf{y}_{k}^{\mathrm{m}}, \mathbf{y}_{k+1}^{\mathrm{m}}, \ldots, \mathbf{y}_{\bar{k}}^{\mathrm{m}}$ in the noncausal case. The sets $\mathbb{X}_{k}$ and $\mathbb{Y}_{k}$ are deduced from prior information, or from the measurements $\mathbf{y}_{k}^{\mathrm{m}}$ via the interpretation function $\phi_{\mathbf{y}}$. In absence of specific prior information, the prior sets $\mathbb{X}_{0}, \ldots, \mathbb{X}_{\bar{k}}$ are all taken as $\mathbb{R}^{n}$ and the prior sets $\mathbb{Y}_{1}, \ldots, \mathbb{Y}_{\bar{k}}$ are all taken as $\mathbb{R}^{\operatorname{dim} \mathbf{y}}$. 
The SCSP $\mathcal{H}=(\mathcal{V}, \mathcal{D}, \mathcal{C})$ associated with the state estimator is defined by

$$
\begin{aligned}
& \mathcal{V}=\left\{\mathbf{x}_{0}, \ldots, \mathbf{x}_{\bar{k}}, \mathbf{y}_{1}, \ldots, \mathbf{y}_{\bar{k}}\right\} \\
& \mathcal{D}=\left\{\mathbb{X}_{0}, \ldots, \mathbb{X}_{\bar{k}}, \mathbb{Y}_{1}, \ldots, \mathbb{Y}_{\bar{k}}\right\} \\
& \mathcal{C}=\left\{\mathbf{x}_{\ell}=\mathbf{f}_{\ell}\left(\mathbf{x}_{\ell-1}\right) ; \ell=1, \ldots, \bar{k}\right\} \cup\left\{\mathbf{y}_{\ell}=\mathbf{g}_{\ell}\left(\mathbf{x}_{\ell}\right) ; \ell=1, \ldots, \bar{k}\right\}
\end{aligned}
$$

Its graph is represented on Figure 6. Despite the presence of arrows, this graph is not oriented. The arrows are only meant to indicate the direction along which the associated function operates.

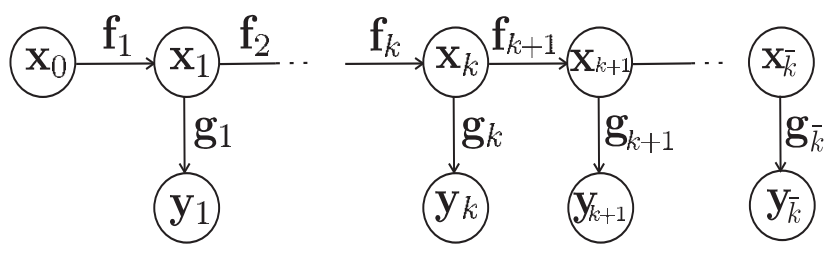

Figure 6: Graph associated with the autonomous system.

In the causal case, the generalized set estimator presented in Section 1 specializes into the following algorithm, where CSE stands for Causal State Estimator.

\section{CSE}

Input: $\mathbb{X}_{0}, \ldots, \mathbb{X}_{\bar{k}}, \mathbb{Y}_{1}, \ldots, \mathbb{Y}_{\bar{k}}$

Initialization:

$1 \quad$ for $\ell:=1$ to $\bar{k},\left\{\mathbb{X}_{\ell}:=\mathbb{X}_{\ell} \cap \mathbf{f}_{\ell}\left(\mathbb{X}_{\ell-1}\right) \cap \mathbf{g}_{\ell}^{-1}\left(\mathbb{Y}_{\ell}\right) ;\right\}$;

$2 \quad$ for $\ell:=\bar{k}$ down to $1,\left\{\mathbb{Y}_{\ell}:=\mathbf{g}_{\ell}\left(\mathbb{X}_{\ell}\right) ; \mathbb{X}_{\ell-1}:=\mathbb{X}_{\ell-1} \cap \mathbf{f}_{\ell}^{-1}\left(\mathbb{X}_{\ell}\right) ;\right\}$;

$3 \quad k:=1$;

Iteration $k$

$4 \quad$ wait for $\mathbf{y}_{k}^{\mathrm{m}}$;

$5 \quad \mathbb{Y}_{k}:=\mathbb{Y}_{k} \cap \phi_{\mathbf{y}}\left(\mathbf{y}_{k}^{\mathrm{m}}\right) ;$

$6 \quad \mathbb{X}_{k}:=\mathbb{X}_{k} \cap \mathbf{g}_{k}^{-1}\left(\mathbb{Y}_{k}\right) ;$

$7 \quad$ for $\ell:=k+1$ to $\bar{k},\left\{\mathbb{X}_{\ell}:=\mathbb{X}_{\ell} \cap \mathbf{f}_{\ell}\left(\mathbb{X}_{\ell-1}\right) ; \mathbb{Y}_{\ell}:=\mathbf{g}_{\ell}\left(\mathbb{X}_{\ell}\right) ;\right\}$;

8 for $\ell:=k$ down to $1,\left\{\mathbb{Y}_{\ell}:=\mathbf{g}_{\ell}\left(\mathbb{X}_{\ell}\right) ; \mathbb{X}_{\ell-1}:=\mathbb{X}_{\ell-1} \cap \mathbf{f}_{\ell}^{-1}\left(\mathbb{X}_{\ell}\right) ;\right\}$;

$9 \quad$ if $(k<\bar{k})\{k:=k+1$; go to Step 4$\}$.

CSE is a specialization of the generalized set estimator presented in Section 1. It performs an optimal contraction during the initialization and after each measurement collection as stated by the following theorem.

Theorem 7 After Step 2 and after each execution of Step 8 of CSE, $\mathcal{H}$ is minimal. 
Proof: Step 1 corresponds to $\operatorname{Fall}(\mathcal{H})$, where $\mathcal{H}=\left(\mathcal{V}, \mathcal{D}, \mathcal{C}, \mathbf{x}_{\bar{k}}\right)$ and Step 2 corresponds to $\operatorname{CLimb}(\mathcal{H})$. From Theorem 6 , these two steps produce the optimal contraction of $\mathcal{H}$. At Step 5 , an external contraction of $\mathcal{H}$ takes place. After Step $5, \mathcal{H}$ is thus no longer minimal, but it is still up-consistent, if we consider $\mathbf{y}_{k}$ as the new root of $\mathcal{H}$. Steps 6,7 and 8 correspond to $\operatorname{Climb}(\mathcal{H})$. After Step 8, $\mathcal{H}$ is minimal from Theorem 5 .

In many practical situations, we are not interested in all variables but only in a few of them. In the context of state estimation, at time $k$, we may only want to estimate $\mathbf{x}_{k}$ and $\mathbf{y}_{k}$. Define the RCSE algorithm, for Recursive CSE, by replacing Steps 6, 7 and 8 in CSE by

$$
\begin{array}{ll}
6 & \mathbb{X}_{k}:=\mathbb{X}_{k} \cap \mathbf{f}_{k}\left(\mathbb{X}_{k-1}\right) \cap \mathbf{g}_{k}^{-1}\left(\mathbb{Y}_{k}\right) \\
7 & \mathbb{Y}_{k}:=\mathbb{Y}_{k} \cap \mathbf{g}_{k}\left(\mathbb{X}_{k}\right)
\end{array}
$$

Note that Step 8 does not exist anymore in RCSE. The following theorem shows that, although much simpler and much more efficient, RCSE provides the same accuracy on $\mathbf{x}_{k}$ and $\mathbf{y}_{k}$ as CSE.

Theorem 8 After Step 2 and after Step 7 of RCSE, $\mathbf{x}_{k}$ and $\mathbf{y}_{k}$ are $\mathcal{H}$-consistent.

Proof: The consistency of $\mathbf{x}_{k}$ and $\mathbf{y}_{k}$ after Step 2 is a direct consequence of the minimality of $\mathcal{H}$ (see Theorem 7). Assume that Theorem 8 is true for $k-1$. Consider $\mathbf{x}_{k}$ as the root of $\mathcal{H}$. $(i)$ Because $\mathcal{H}_{\mathbf{x}_{k-1}}=\operatorname{SubTree}\left(\mathcal{H}, \mathbf{x}_{k-1}\right)$ is up-consistent before Step 4, it remains so after Step 5. (ii) Because $\mathcal{H}_{\mathbf{x}_{k+1}}=\operatorname{SubTree}\left(\mathcal{H}, \mathbf{x}_{k+1}\right)$ is up-consistent after Step 2, it is also up-consistent after Step 5. (iii) Because $\mathcal{H}_{\mathbf{y}_{k}}=\operatorname{SubTree}\left(\mathcal{H}, \mathbf{y}_{k-1}\right)$ is a leaf, it is up-consistent. From $(i)$, (ii) and (iii) and from Theorem 1, after Step 6 of RCSE, $\mathcal{H}$ is up-consistent and thus its root $\mathbf{x}_{k}$ is $\mathcal{H}$-consistent. From Theorem $3, \mathbf{y}_{k}$ is $\mathcal{H}$-consistent after Step 7.

Remark 2 RCSE is similar to the algorithm proposed in [11], [12]. The main difference is that in RCSE the initialization steps make it possible to take into account some prior information on the future state or output vectors. Moreover, the use of the CSP theory made it possible to derive Theorem 8 in a very simple way.

Consider now the noncausal case. Assume that the $\bar{k}$ domains $\mathbb{Y}_{k}, k \in\{1, \ldots, \bar{k}\}$ are available. The minimal contraction of $\mathcal{H}$ is computed using FALL-ClimB, where the root is chosen as $\mathbf{x}_{\bar{k}}$. It can be translated, for this special problem, into the following NCSE algorithm, for Non Causal State Estimator 


\section{NCSE}

Input: $\mathbb{X}_{0}, \ldots, \mathbb{X}_{\bar{k}}, \mathbb{Y}_{1}, \ldots, \mathbb{Y}_{\bar{k}}$

Initialisation:

$1 \quad$ For $k:=1$ to $\bar{k}, \mathbb{Y}_{k}:=\mathbb{Y}_{k} \cap \phi_{\mathbf{y}}\left(\mathbf{y}_{k}^{\mathrm{m}}\right)$;

$2 \quad$ For $k:=1$ to $\bar{k},\left\{\mathbb{X}_{k}:=\mathbb{X}_{k} \cap \mathbf{f}_{k}\left(\mathbb{X}_{k-1}\right) \cap \mathbf{g}_{k}^{-1}\left(\mathbb{Y}_{k}\right) ;\right\}$;

$3 \quad$ For $k:=\bar{k}$ down to $1,\left\{\mathbb{Y}_{k}:=\mathbf{g}_{k}\left(\mathbb{X}_{k}\right) ; \mathbb{X}_{k-1}:=\mathbb{X}_{k-1} \cap \mathbf{f}_{k}^{-1}\left(\mathbb{X}_{k}\right) ;\right\}$;

Step 2 corresponds to FALL and Step 3 corresponds to CLIMB. Note that the contracted domain $\mathbb{Y}_{k}$ contains the actual output vector $\mathbf{y}_{k}^{*}$ with a better accuracy than the former domain.

\section{Test-case}

Consider the nonlinear system

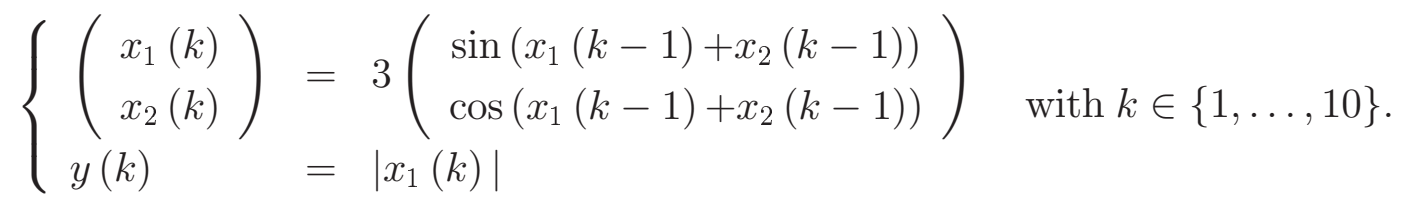

For $\mathbf{x}^{*}(0)=\left(\begin{array}{ll}0 & 0\end{array}\right)^{\mathrm{T}}$, the values $\mathbf{x}^{*}(k)$ and $y^{*}(k), k \in\{1, \ldots, 10\}$, have been generated by simulation of this system. The measurements $y^{\mathrm{m}}(k)$ have then been obtained by adding a bounded noise to the actual output $y^{*}(k)$ :

$$
y^{\mathrm{m}}(k)=y^{*}(k)+n(k),
$$

where $n(k)$ is a random variable with a uniform distribution in the interval $[-0.1,0.1]$. The measurement uncertainty sets are taken as

$$
\mathbb{Y}(k)=\phi_{\mathbf{y}(k)}\left(y^{\mathrm{m}}(k)\right)=y^{\mathrm{m}}(k)+[-0.1 ; 0.1]
$$

Note that the condition

$$
y^{*}(k) \in \mathbb{Y}(k)
$$

is satisfied for all $k$. The domains obtained for $\mathbf{x}(k)$ by RCSE and NCSE are depicted in Figure 7. The total computing time for RCSE and NCSE is less than one minute on a Pentium 133 $\mathrm{MHz}$ personnal computer. The frames of all subfigures are $[-4,4] \times[-4,4]$. The initial domains given to the estimators are $\mathbb{X}(0)=\cdots=\mathbb{X}(10)=\mathbb{R}^{2}$ and $\mathbb{Y}(1)=\cdots=\mathbb{Y}(10)=\mathbb{R}$. The first subfigure is entirely grey, which means that RCSE is unable to provide any information about $\mathbf{x}(0)\left(\mathbb{X}(0)=\mathbb{R}^{2}\right)$, contrary to the non causal estimator. The last two subfigures, for $k=10$, are identical because both estimators have now processed the same information. 


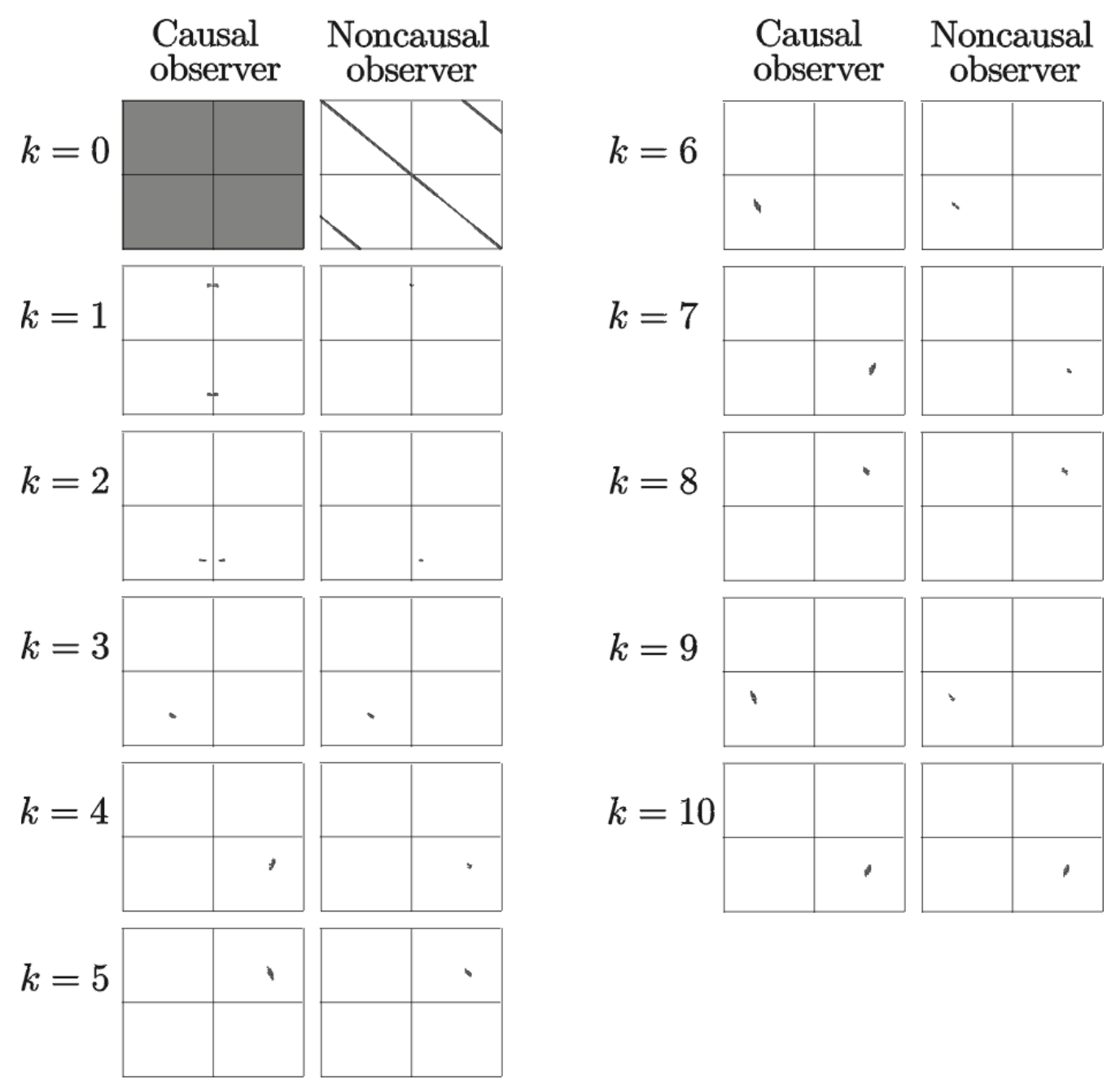

Figure 7: Causal and noncausal set observations

Remark 3 The computer implementation of FALL and CLIMB requires a representation for sets and an implementation of the local contraction operator $\rho_{i}()$. A domain $\mathbb{V}$ is represented by a subpaving (union of boxes) that encloses it. In our context, the local contraction operator $\rho_{i}()$ corresponds either to the image $\mathbf{f}(\mathbb{V})$ of a set $\mathbb{V}$ by a vector function $\mathbf{f}$ or to the reciprocal image $\mathbf{f}^{-1}(\mathbb{W})$ of a set $\mathbb{W}$ by a vector function $\mathbf{f}$. The computation of a guaranteed enclosure of $\mathbf{f}(\mathbb{V})$ can be performed by the algorithm IMAGESP [11] and a guaranteed enclosure of $\mathbf{f}^{-1}(\mathbb{W})$ can be performed by the algorithm SIVIA [6]. These two algorithms are based on interval analysis [17].

\section{Conclusions and perpectives}

Nonlinear parameter and state estimation has been cast into the general framework of SCSPs. The notion of SCSP is itself a generalization to the vector case of that of ICSP (Interval 
Constraint Satisfaction Problem) rather classical in the area of artificial intelligence [5]. The main difference between SCSPs and ICSPs is that ICSPs handle intervals whereas SCSPs handle subsets of $\mathbb{R}^{n}$. This made it possible to obtain two powerful theorems (the propagation and the retropropagation theorems) that are not true in an ICSP context. Existing algorithms [1], have been generalized to SCSPs whose graphs are trees, under the names of FALL and CLIMB. Contrary to what happens for ICSPs, we have shown that a single execution of FALL followed by a single execution of CLIMB was sufficient to produce an optimal contraction.

In the special case of causal state estimation, the algorithms proposed in [11] and [12], can be interpreted in the framework of the more general FALL-CLIMB algorithm.

We have chosen in this paper not to put emphasis on computer implementation, but this is of course a critical issue, addressed in detail in [10]. The source code in $\mathrm{C}++$ Builder 4, and an executable program for IBM-compatible PCs corresponding to the example are available on request.

\section{References}

[1] F. Benhamou, F. Goualard, L. Granvilliers, and J. F. Puget. Revising hull and box consistency. In International Conference on Logic Programming, pages 230-244, Las Cruces, USA, 1999. MIT Press.

[2] J. C. Cleary. Logical arithmetic. Future Computing Systems, 2(2):125-149, 1987.

[3] E. Davis. Constraint propagation with interval labels. Artificial Intelligence, 32:281-331, 1987.

[4] A. Gelb. Applied Optimal Estimation. MIT Press, Cambridge, 1974.

[5] E. Hyvonen. Constraint reasoning based on interval arithmetic; the tolerance propagation approach. Artificial Intelligence, 58:71-112, 1992.

[6] L. Jaulin and E. Walter. Set inversion via interval analysis for nonlinear bounded-error estimation. Automatica, 29(4):1053-1064, 1993.

[7] R. Kalman. A new approach to linear filtering and prediction problems. Trans. of AMSE, Part D, J. of Basic Engineering, 82:35-45, 1960.

[8] W. Kang and A. Krener. Nonlinear observer design, a backstepping approach. Preprint, Mathematics, UC Davis, 1998. 
[9] M. Kendall and A. Stuart. The Advanced Theory of Statistics, Vol. 2, Inference and Relationship, 2nd Edition. Charles Griffin and Company, London, 1967.

[10] M. Kieffer. Estimation ensembliste par analyse par intervalles, application à la localisation d'un véhicule. PhD dissertation, Université Paris-Sud, Orsay, 1999.

[11] M. Kieffer, L. Jaulin, and E. Walter. Guaranteed recursive nonlinear state estimation using interval analysis. In Proc. 37th IEEE Conference on Decision and Control, pages 3966-3971, Tampa, Florida, December 16-18, 1998.

[12] M. Kieffer, L. Jaulin, E. Walter, and D. Meizel. Guaranteed mobile robot tracking using interval analysis. In Proc. MISC'99 Workshop on Application of Interval Analysis to Systems and Control, pages 347-359, Girona, February 24-26, 1999.

[13] Y. Landau. Adaptive Control, The Model Reference Approach. Marcel Dekker, New York, 1979.

[14] D. Luenberger. Observers for multivariable systems. IEEE Transactions on Automatic Control, 11:190-197, 1966.

[15] M. Milanese, J. Norton, H. Piet-Lahanier, and E. Walter (Eds). Bounding Approaches to System Identification. Plenum Press, New York, 1996.

[16] M. Milanese and A. Vicino. Estimation theory for nonlinear models and set membership uncertainty. Automatica, 27:403-408, 1991.

[17] R. E. Moore. Methods and Applications of Interval Analysis. SIAM Publ., Philadelphia, 1979.

[18] J. P. Norton (Ed.). Special issue on bounded-error estimation: Issue 1. Int. J. of Adaptive Control and Signal Processing, 8(1):1-118, 1994.

[19] J. P. Norton (Ed.). Special issue on bounded-error estimation: Issue 2. Int. J. of Adaptive Control and Signal Processing, 9(1):1-132, 1995.

[20] H. Sorenson (Ed.). Special issue on applications of Kalman filtering. IEEE Transactions on Automatic Control, 28(3):253-434, 1983.

[21] E. Walter (Ed.). Special issue on parameter identifications with error bounds. Mathematics and Computers in Simulation, 32(5\&6):447-607, 1990.

[22] D. Waltz. The Psychology of Computer Vision, chapter Generating semantic descriptions from drawings of scenes with shadows, in P.H. Winston (ed.), pages 19-91. McGraw-Hill, New-York, 1975. 